\title{
The Electrical Characteristics Model of GaN/InGaN/GaN Heterostructure in InGaN-based LED
}

\author{
Budi Mulyanti $^{1}$, Lilik Hasanah ${ }^{2} \&$ Khairurrijal $^{3}$ \\ ${ }^{1}$ Department of Electrical Engineering Education, Faculty of Technology and Vocational Education, Indonesia \\ University of Education (UPI), Jl. Dr. Setiabudhi No 207, Bandung 40135, Indonesia \\ ${ }^{2}$ Department of Physics Education, Faculty of Mathematics and Natural Sciences Education, Indonesia \\ University of Education (UPI), Jl. Dr. Setiabudhi No 229, Bandung 40135, Indonesia \\ ${ }^{3}$ Physics of Electronic Materials Research Division, Faculty of Mathematics and Natural Sciences, Bandung \\ Institute of Technology, J1. Ganesha 10, Bandung 40132, Indonesia \\ Correspondence: Budi Mulyanti, Department of Electrical Engineering Education, Faculty of Technology and \\ Vocational Education, Indonesia University of Education (UPI), Jl. Dr. Setiabudhi No 207, Bandung 40135, \\ Indonesia. E-mail: b_mulyanti@yahoo.com
}

Received: February 3, $2012 \quad$ Accepted: February 17, $2012 \quad$ Online Published: May 1, 2012

doi:10.5539/apr.v4n2p98

URL: http://dx.doi.org/10.5539/apr.v4n2p98

\begin{abstract}
The calculation model of tunneling current through GaN/InGaN/GaN heterostructure in InGaN-based LED using the transfer matrix method employed to verify the result of calculation of tunneling current implemented analytically. The analytical method applied through solving theoretically the Schrödinger equation, whereas, the transfer matrix method divided the solution area into a less size of $\mathrm{N}$ segment compared to the observed potential width size, where the potential energy of each segment was assumed constant. Verification employed to the thickness of depletion region and bias voltage variations. The obtained result has shown that the analytical result of calculation simmilar with the calculation result using transfer matrix method. The calculation model was then extended to calculate the tunneling current for different temperature.
\end{abstract}

Keywords: tunneling current, GaN/InGaN structure, InGaN based LED, TMM

\section{Introduction}

At present, microelectronic devices have better developed with their more amazing performance. The increase of the device performance has been triggered by the smaller size device. Effort in minimizing the size of the device and increasing the performance of the device will surely one day reach its stagnancy, especially when the device cannot be minimized any longer, whereas the requirement to increase its performance should then be fulfilled. To solve such problem, nowadays, the scope of the research material as main component device has been directed to the searching of the new material that its characteristics are compatible to the device through simulating the material-based devise characteristics, so the characteristics of the device can be predicted beforehand without first fabricating the device. It will, of course, be more efficient and effective to be directed to the effort of finding and searching new material.

Since 1999, InGaN-based LED's have gained great attention and provide the impressive results due to their highest external quantum efficiencies of $18 \%$ and $20 \%$ at low currents of $0.6 \mathrm{~mA}$ and $0.1 \mathrm{~mA}$, respectively (Mukai et al, 1999). Although InGaN-based LEDs are already commercially available for visible light emission such as in display and automotive lighting applications, further improvement of the light output power and the external quantum efficiency are required (Chu et al., 2010). To find out the efficiency of LEDs, it would require the study of electrical characteristics in advance. The electrical characteristics of LEDs can be obtained by examining the $p-n$ junction of heterostructure. In this study, the abrupt $p-n$ junction is used by assuming that all dopants are fully ionized. Barriers of an abrupt $\mathrm{p}-\mathrm{n}$ heterojunction are greater than that of the graded junction due to the formation of electron barrier at the abrupt junction. Furthermore, electrical characteristics of the LEDs are conducted to determine the leakage current that occurs at high injection current density (Polyakov et al., 2004; Schubert et al., 2006).

Study of the theory of electrical properties of $\mathrm{GaN} / \mathrm{InGaN} / \mathrm{GaN}$ heterostructure in InGaN based-LED theoretically can be performed using carrier transport model such as diffusion-drift transport, energy transport, 
hydrodynamic, and quantum transport models. However, the most common model used is diffusion-drift transport model (Pejcinovic et al., 1989).

With the shrinking of the devices size, the electron transport due to diffusion and drift becomes smaller than that of the quantum transport, therefore the diffusion-drift transport can be ignored in the calculations and in other word, only quantum transport included in the calculation. Quantum transport in the heterojunction structure can be determined by solving Schrödinger equation. There are two kinds of methods can be used to solve the Schrödinger equation, those are analytical (Lee, 1993) and semi-numerical methods (Mao, 2007). And it has been proved that the transfer matrix method (TMM) is more accurate than the conventional finite difference methods (Abdolkader et al., 2004). To examine the analytical formula obtained, in this paper the tunneling current through $\mathrm{GaN} / \mathrm{InGaN} / \mathrm{GaN}$ heterostructure in InGaN-based LED was also calculated using TMM.

\section{Theoretical Model}

\subsection{Tunneling Current Calculation Using Analytical Methods}

The Schrödinger equation that should be solved to obtain the behavior of electron in the anisotropic heterostructure is (Hasanah, 2008):

$$
H \psi(\mathbf{r})=E \psi(\mathbf{r}) .
$$

$H$ is Hamiltonian namely:

$$
H=\left(\frac{1}{2 m_{\perp}(z)} \hat{p}_{\perp}^{2}+\frac{1}{2 m_{z}(z)} \hat{p}_{z}^{2}+U(z)\right),
$$

$\mathrm{m}_{\perp}$ and $\mathrm{m}_{\mathrm{z}}$ are effective mass of the electron perpendicular and parallel to the interface, respectively, $\mathbf{p}$ is momentum vector and $\mathrm{U}(\mathbf{r})$ is potential energy. Figure 1 shows the potential profile in z-direction. The electrons come from region I toward potential barrier (in region II) where the effective mass of electron only depend on the z-direction. The wave function in Eq. (1) is (Hasanah, et al., 2008):

$$
\psi(\mathbf{r})=\varphi(z) \exp (-i \gamma z) \exp \left(i\left(k_{x} x+k_{y} y\right)\right)
$$

The wave function in Eq. (3) satisfies the following one-dimensional Schrödinger equation:

$$
\left(\frac{1}{2 m_{\perp}(z)} \hat{p}_{\perp}^{2}+\frac{1}{2 m_{z}(z)} \hat{p}_{z}^{2}+U(z)\right) \psi=E \psi
$$

where:

$$
E_{z}^{G a N}=E-\frac{\hbar^{2} k_{r}^{2}}{2 m_{\perp-G a N}^{*}}
$$

is the energy of incident electron in z-direction, $\vec{k}_{r}$ and $\vec{r}$ are the wave vector and the transverse coordinate, respectively, of the electron in the plane parallel to the interface, $m_{\perp-G a N}^{*}$ is effective mass of electron perpendicular to the interface in the $\mathrm{GaN}$ region.

The longitudinal energy of electron in the $\mathrm{InGaN}$ region then can be written as:

$$
E_{z}^{\operatorname{InGaN}}=E-\frac{\hbar^{2} k_{r}^{2}}{2 m_{\text {In GaN }}^{*}}
$$

$m_{\text {InGaN }}^{*}$ effective mass of electron parallel to the interface in the $\mathrm{InGaN}$ region.

While the total energy of tunneling electron has to be constant, therefore:

$$
E_{z}^{I n G a N}=E_{z}^{s}+\frac{\hbar^{2} k_{r}^{2}}{2 m_{\perp-G a N}^{*}}-\frac{\hbar^{2} k_{r}^{2}}{2 m_{I n G a N}^{*}}
$$

In the $\mathrm{GaN}$ and $\mathrm{InGaN}$ regions, Schrödinger equation along the tunneling direction can be written as:

$$
\begin{aligned}
& {\left[-\frac{\hbar^{2}}{2 m_{z-G a N}^{*}} \frac{\partial^{2}}{\partial z^{2}}+U(z)\right] \phi(z)=E_{z}^{G a N} \phi(z)} \\
& {\left[-\frac{\hbar^{2}}{2 m_{\text {InGaN }}^{*}} \frac{\partial^{2}}{\partial z^{2}}+U(z)\right] \phi(z)=E_{z}^{I n G a N} \phi(z)}
\end{aligned}
$$




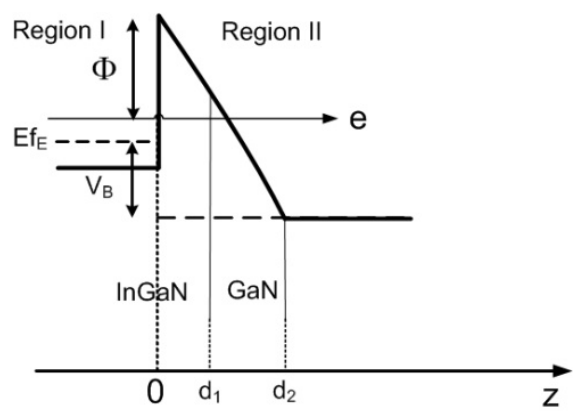

Figure 1. Energy-band diagram of InGaN / GaN abrupt p-n junction

From Eq. (3), (4) and (5), it can be obtained:

$$
\left[-\frac{\hbar^{2}}{2 m_{\text {InGaN }}^{2}} \frac{\partial^{2}}{\partial z^{2}}+\left(U(z)-\frac{\hbar^{2} k_{r}^{2}}{2 m_{\perp-G a N}^{*}}\left(1-\frac{m_{\perp-G a N}^{*}}{m_{I n G a N}^{*}}\right)\right)\right] \phi(z)=E_{z}^{G a N} \phi(z)
$$

\subsection{Calculation of Tunneling Current Using Transfer Matrix Method (TMM)}

The transmission coefficient can be calculated using transfer matrix method (TMM). The TMM is a very common semi-numerical method in Physisc and Mathematics to solve the problem when the total system can be divided into $\mathrm{N}$ subsystems that interact only with neighboring subsystems. In this case, the TMM is used to solve the Schrödinger equation when the solution region of potential energy is divided into $\mathrm{N}$ segments. The width of each segment much smaller than that of the actual potential, while the potential energy in each segment is assumed to be constant as shown in Figure 2. If the number of segments increase, the approximation potential will reach the actual potential. The Schrödinger equation of each segment could be solved through applying the exponential function, that then arranged in a set of matrices that contain the boundary conditions at each segment interface. The transmission coefficient is then calculated from the set of matrices. The calculation is done for the abrupt p-n heterojunction, where its resistance is greater than that of the graded junction due to the existence of electron barrier formed by abrupt junction, as shown in Figure 2. The solid line shows the actual potential profile while the dotted line represents the approximation potential used to solve the Schrödinger equation using the TMM.

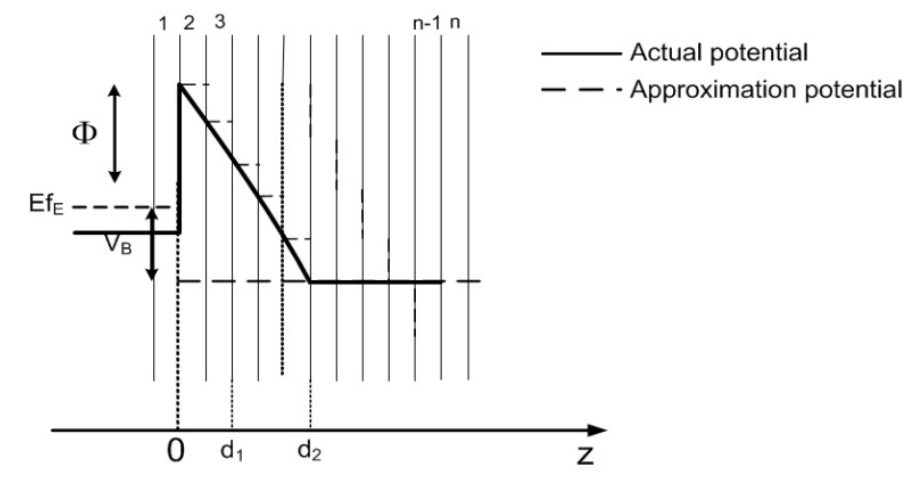

Figure 2. The GaN/InGaN potential profile divided into $\mathrm{N}$ segments that applied in the calculation of the coefficient transmission using transfer matrix method (TMM)

The wave function of electron regardless to the time of each area that become the solution of Schrödinger equation of the approximation potential as many as $\mathrm{N}$ segments can be written as:

$$
\begin{array}{lr}
\psi_{1}(z)=\left(A_{1} e^{i k_{1} z}+B_{1} e^{-i k_{1} z}\right) e^{-i \gamma_{1} z} e^{-i\left(k_{x} x+k_{y} y\right)} & \mathrm{z} \leq 0, \\
\psi_{j}(z)=\left(A_{m} e^{k_{m} z}+B_{m} e^{-k_{m} z}\right) e^{-i \gamma 2 z} e^{-i\left(k_{x} x+k_{y} y\right)} & 0<\mathrm{z}<\mathrm{d}_{1},
\end{array}
$$




$$
\psi_{N}(z)=\left(A_{N} e^{i k_{3} z}+B_{N} e^{-i k_{3} z}\right) e^{-i \gamma_{1} z} e^{-i\left(k_{x} x+k_{y} y\right)} \quad \mathrm{d}_{1}<\mathrm{z}<\mathrm{d}_{2}
$$

where $A_{1}, B_{1}, A_{m}, B_{m}, A_{N}$ and $B_{N}$ are constants, with $m=2, \ldots, N-1$.

To simplify the calculation, the constant value of $A_{1}$ is taken as 1 , and $B_{N}$ is zero, because it is assumed that there is no reflection in the interface of segment. The wave number $\mathrm{k}_{\mathrm{m}}$ for the region $0<\mathrm{z}<\mathrm{d}_{1}$, is stated as follows:

$$
k_{m}^{2}=\frac{2 m_{1}}{\hbar^{2}} \Phi-k_{1}^{2},
$$

whereas the wave number, $k_{m}$ for the decrease of potential barrier, on $\mathrm{d}_{1}<\mathrm{z}<\mathrm{d}_{2}$, is

$$
k_{m}^{2}=\frac{2 m_{0}}{\hbar^{2}} \frac{1}{\alpha_{z z, 2}}\left(\Phi-e \frac{\Phi+V_{B C}}{d_{2}} z\right)-\frac{\alpha_{z z, 1}}{\alpha_{z z, 2}} k_{1}^{2} .
$$

The boundary conditions and continuity at each segment of the interface have to be applied. If there is $\mathrm{N}$ number of segment, then the number of interface points are (N-1) items, hence the number of boundary conditions are $2(N-1)$ items.

The result of application of boundary condition at the point of $z_{l}$ is:

$$
\begin{aligned}
& \left(\begin{array}{cc}
e^{i\left(k_{1}-\gamma_{1}\right) z_{1}} & e^{-i\left(k_{1}+\gamma_{1}\right) z_{1}} \\
a_{1} e^{i\left(k_{1}-\gamma_{1}\right) z_{1}} & -a_{1} e^{-i\left(k_{1}+\gamma_{1}\right) z_{1}}
\end{array}\right)\left(\begin{array}{c}
1 \\
B_{1}
\end{array}\right)= \\
& \left(\begin{array}{cc}
e^{\left(k_{2}-\gamma_{2}\right) z_{1}} & e^{-i\left(k_{2}+\gamma_{2}\right) z_{1}} \\
a_{2} e^{i\left(k_{2}-\gamma_{2}\right) z_{1}} & -a_{2} e^{-i\left(k_{2}+\gamma_{2}\right) z_{1}}
\end{array}\right)\left(\begin{array}{l}
A_{2} \\
B_{2}
\end{array}\right),
\end{aligned}
$$

where $a_{1}=\frac{i k_{1}}{m_{1}}$ and $a_{2}=\frac{k_{2}}{m_{2}}$.

The result of application of boundary condition at the point of $z_{2}$ to $z_{N-2}$ is:

$$
\begin{aligned}
& \left(\begin{array}{cc}
e^{i\left(k_{m}-\gamma_{m}\right) z_{m}} & e^{-i\left(k_{m}+\gamma_{m}\right) z_{m}} \\
a_{m} e^{i\left(k_{m}-\gamma_{m}\right) z_{m}} & -a_{m} e^{-i\left(k_{m}+\gamma_{m}\right) z_{m}}
\end{array}\right)\left(\begin{array}{c}
A_{m} \\
B_{m}
\end{array}\right)= \\
& \left(\begin{array}{cc}
e^{\left(k_{m+1}-\gamma_{m+1}\right) z_{m}} & e^{-i\left(k_{m+1}+\gamma_{m+1}\right) z_{m}} \\
a_{m+1} e^{i\left(k_{m+1}-\gamma_{m+1}\right) z_{m}} & -a_{m+1} e^{-i\left(k_{m+1}+\gamma_{m+1}\right) z_{m}}
\end{array}\right)\left(\begin{array}{c}
A_{m+1} \\
B_{m+1}
\end{array}\right),
\end{aligned}
$$

where $a_{m}=\frac{k_{m}}{m_{z z m}}$ and $a_{m+1}=\frac{k_{m+1}}{m_{z z m+1}}$.

And the result of application of boundary condition at the point of $z_{N-1}$ is:

$$
\begin{aligned}
& \left(\begin{array}{cc}
e^{i\left(k_{N-1}-\gamma_{N-1}\right) z_{N-1}} & e^{-i\left(k_{N-1}+\gamma_{N-1}\right) z_{N-1}} \\
a_{N-1} e^{i\left(k_{N-1}-\gamma_{N-1}\right) z_{N-1}} & -a_{N-1} e^{-i\left(k_{N-1}+\gamma_{N-1}\right) z_{N-1}}
\end{array}\right)\left(\begin{array}{c}
A_{N-1} \\
B_{N-1}
\end{array}\right)= \\
& \left(\begin{array}{cc}
e^{\left(k_{N}-\gamma_{N}\right) z_{N-1}} & e^{-i\left(k_{N}+\gamma_{N}\right) z_{N-1}} \\
a_{N} e^{i\left(k_{N}-\gamma_{N}\right) z_{N-1}} & -a_{N} e^{-i\left(k_{N}+\gamma_{N}\right) z_{N-1}}
\end{array}\right)\left(\begin{array}{c}
A_{N} \\
0
\end{array}\right),
\end{aligned}
$$

where $a_{N-1}=\frac{k_{N-1}}{m_{z z N-1}}$ and $a_{N}=\frac{i k_{N}}{m_{z z N}}$.

From Eq. (16)-(14), it can be obtained the following equation:

$$
\left(\begin{array}{c}
1 \\
B_{1}
\end{array}\right)=\left(\begin{array}{ll}
a_{11} & a_{12} \\
a_{21} & a_{22}
\end{array}\right)\left(\begin{array}{c}
A_{N} \\
0
\end{array}\right),
$$

And electron transmission coefficient, $t$ can be calculated from 


$$
t=A_{N}=\frac{1}{a_{11}} .
$$

While the electron transmittance $T$ can be obtained from:

$$
T=t^{*} t
$$

Where $t^{*}$ is the conjugate of transmission coefficient, $t$.

Tunneling current can be calculated using equation below (Khairurrijal et al., 2006):

$$
J=\int_{0}^{\infty} \frac{q m_{o}}{2 \pi^{2} \hbar^{3}} \frac{1}{\alpha_{z z 1}} T\left(E_{z}\right)\left(\int_{0}^{\infty} f_{L}(E)-f_{R}(E) d E_{x y}\right) d E,
$$

where $T\left(E_{z}\right)$ is the electron transmittance as a function of longitudinal energy $E_{z}$ and $E_{\mathrm{xy}}$ is the transversal energy. The functions of Fermi distribution on the left and the right contacts, are:

$$
f_{L}(E)=\frac{1}{1+\exp \left[\left(E-E_{F L}\right) / k T\right]}
$$

and

$$
f_{R}(E)=\frac{1}{1+\exp \left[\left(E-E_{F R}\right) / k T\right]},
$$

which denote the probability of electron energy, $\mathrm{E}$ is occupied. $E_{F L}$ and $E_{F R}$ each respectively is left and right side fermy energy.

\section{Results and Discussion}

The calculation of transmitance and tunneling current trough abrupt p-n heterojunction of GaN/InGaN in InGaN-based LED has been performed. The discontinuity band between $\mathrm{GaN}$ and InGaN regions is around 0.2 $\mathrm{eV}$, that because of the potential barrier height. The value of electron effective mass in InGaN is higher than that of in $\mathrm{GaN}$, and bias voltage of $\mathrm{V}_{\mathrm{B}}$ is given to the $\mathrm{p}$ - $\mathrm{n}$ junction.

The tunneling current of electrons through abrupt p-n heterojunction of InGaN/GaN with barrier thickness of 10 $\mathrm{nm}$ and $50 \mathrm{~nm}$, and bias voltage $\left(\mathrm{V}_{\mathrm{B}}\right)$ varied from 0 to $100 \mathrm{mV}$ resulted by analytical and semi-numerical method using TMM calculations are shown in Figure 3 and 4, respectively.

The result of tunneling current calculated using the transmittance obtained by analytical method equals to that of semi-numerical method using TMM. Furthermore, Figure 5 shows that the tunneling current of electron tends to decrease by the increase in the potential barrier thickness. This is caused by the increased in effective potential in Eq. (10) that would cause the decrease in transmittance and tunneling current of electron. For this case, the calculation result of tunneling current using analytical transmittance equals to that of that its transmittance was calculated using TMM.

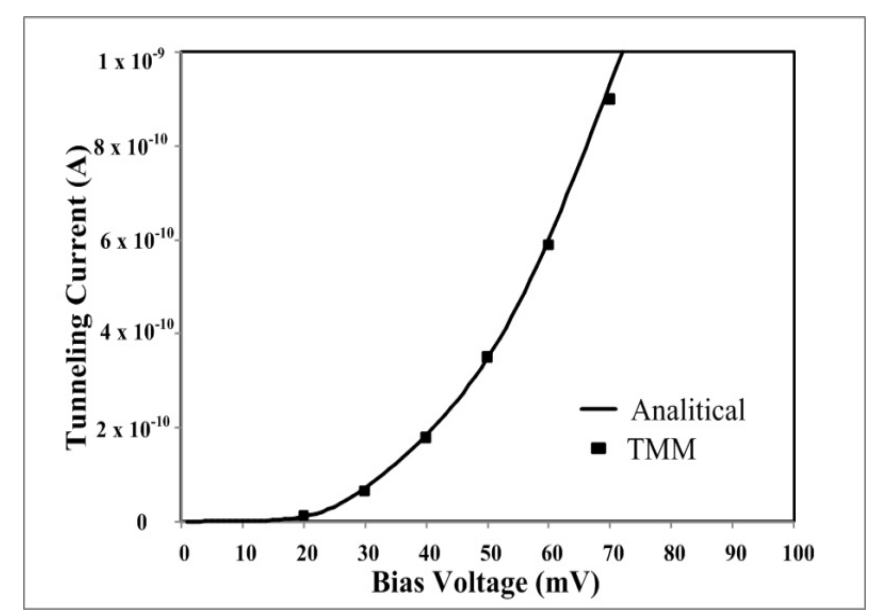

Figure 3. The tunneling current through abrupt p-n heterojunction of InGaN/GaN with the barrier thickness of 10 $\mathrm{nm}$, and bias voltage varied from 0 until $100 \mathrm{mV}$, resulted from analytical and TMM calculation 


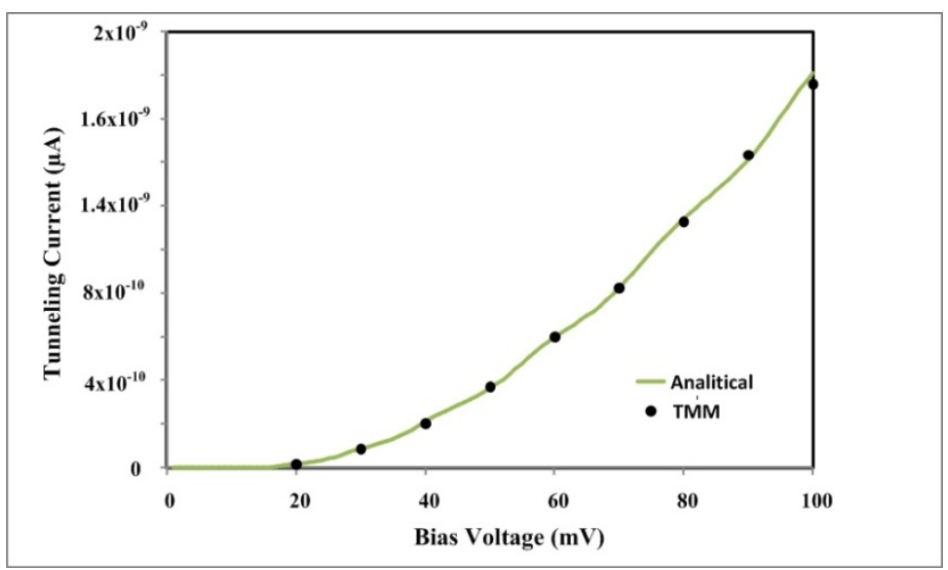

Figure 4. The tunneling current through abrupt p-n heterojunction of $\mathrm{InGaN} / \mathrm{GaN}$ with the barrier thickness of 50 $\mathrm{nm}$, and bias voltage varied from 0 until $100 \mathrm{mV}$, resulted from analytical and TMM calculation

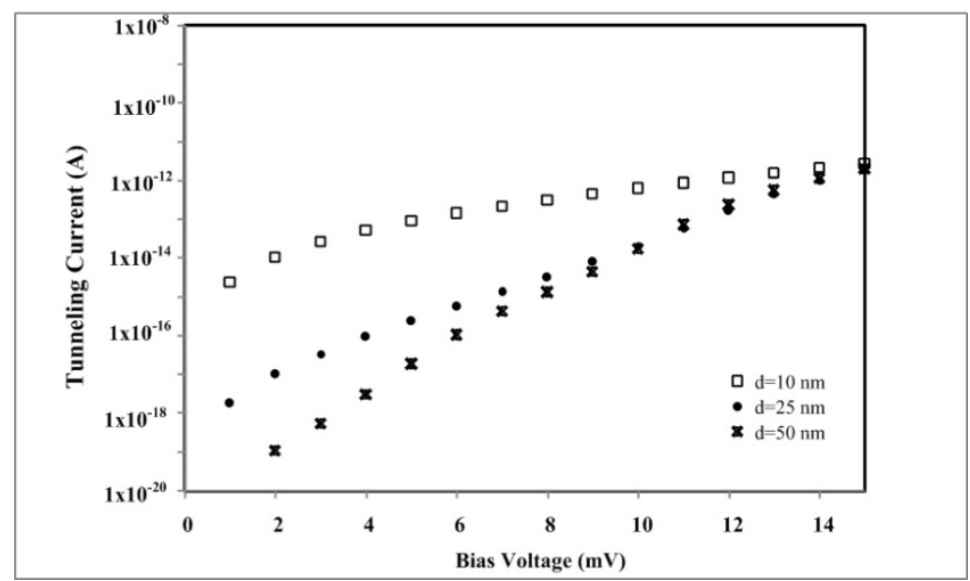

Figure 5. The tunneling current of abrupt p-n heterojunction of $\mathrm{InGaN} / \mathrm{GaN}$ at varied barrier thicknesses of 10, 25 and $50 \mathrm{~nm}$ and bias voltage of 0 to $15 \mathrm{mV}$ resulted from analytical calculation.

The formulation of tunneling current is then applied to calculate the tunneling current in different temperatures as shown in Figure 6. This calculation is also applied in the abrupt p-n heterojunction with varied temperatures of $150 \mathrm{~K}, 300 \mathrm{~K}$ and $500 \mathrm{~K}$ for $\mathrm{V}_{\mathrm{B}}$ varied from 0 to $100 \mathrm{mV}$, and the depletion thickness of $20 \mathrm{~nm}$. From this study, the result shows that the tunneling current tends to decrease by the increase in temperature.

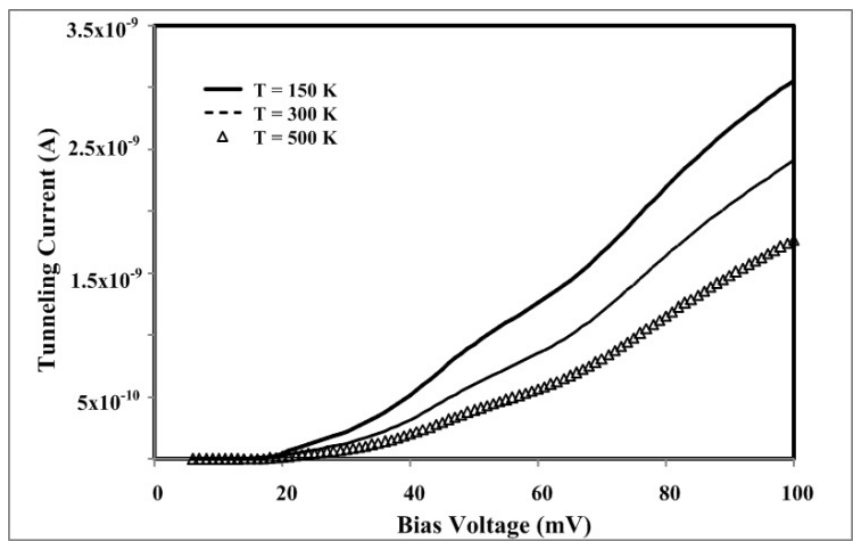

Figure 6. The tunneling current of abrupt p-n heterojunction of InGaN/GaN with varied temperatures of $150 \mathrm{~K}$, $300 \mathrm{~K}$ and $500 \mathrm{~K}$ for depletion thickness of $25 \mathrm{~nm}$ and varied bias voltage from 0 to $100 \mathrm{mV}$ 


\section{Conclusion}

This research employed the calculation of tunneling current through $\mathrm{GaN} / \mathrm{InGaN} / \mathrm{GaN}$ heterostructure in InGaN-based LED by inserting the quantum effect. Tunneling current resulted from the calculation of analytic transmittance equals to that of calculated from the transmittance using the transfer matrix method. The results of the calculation show that the increase in depletion thickness causes the decrease in the tunneling current while the increase in voltage bias causes the increase in the tunneling current. The obtained model was then applied to calculate the tunneling current in different temperature, where the increase in temperature causes the decrease in the tunneling current.

\section{References}

Abdolkader, T. M., Hassan, H. H., Fikry, W., \& Omar, O. A. (2004). Solution of Schrödinger in Double-Gate MOSFETs using Transfer Matrix Method. Electronics Letters, 40, 20. http://dx.doi.org/10.1049/el:20045595

Chu, Y. C., Jin, B. L., Sang, J. L., Sang, H. H., Tae, Y. P., Kim, J. W., Kim, Y. C., \& Seong, J. P. (2010). Improvement of Light Output Power of InGaN/GaN Light-emitting Diode by Lateral Epitaxial Overgrowth Using Pyramidal-shaped $\mathrm{SiO}_{2}$, Optics Express, 18, 2. 1462.

Hasanah, L., Abdullah, M., Sukirno, Winata, T., \& Kharurrijal. (2008). Model of a Tunneling Current in an Anisotropic Si/Sil- $x \mathrm{Ge} x / \mathrm{Si}$ Heterostructure with a Nanometer-thick Barrier Including the Effect of Parallel-Perpendicular Kinetic Energy Coupling. Semicond. Sci. Technol., 23, 125024. http://dx.doi.org/10.1088/0268-1242/23/12/125024

Khairurrijal, Miyazaki, S., \& Hirose, M (1999). Electron Field Emission from a Silicon Subsurface Based on a Generalized Airy Function Approach. $S$ J. Vac. Sci. Technol. B., $17,306$. http://dx.doi.org/10.1116/1.590555

Lee, B. (1993). Electron Tunneling Time through a Heterostructure Potential Barrier, Supperlattices and Microstructures, 14, 295. http://dx.doi.org/10.1006/spmi.1993.1143

Mao, L. (2007). The Effects of the Injection-Channel Velocity on the Gate Leakage Current of Nanoscale MOSFETs. IEEE Electron Device Letters, 28, 161. http://dx.doi.org/10.1109/LED.2006.889214

Mukai, T., Yamada, M., \& Nakamura, S. (1999): Characteristics of InGaN-based UV/Blue/Green/Amber/Red Light-Emitting Diodes. Jpn. J. Appl. Phys, 38, Part 1, No. 7A. 3976-3981.

Pejcinovic, B., Kay, L. E., Tang, T. W., \& Navon, D. H. (1989). Numerical Simulation and Comparison of Si BJTs and $\mathrm{Si}_{1-\mathrm{x}} \mathrm{Ge}_{\mathrm{x}}$ HBTs. IEEE Trans. Electron Devices, 36. 2129. http://dx.doi.org/10.1109/16.40892

Polyakov, A. Y., Smirnov, N. B., Govorkov, A. V., Kim, J., Ren, F., Thaler, G. T., Fraizer, R. M., Gila, B. P., Abernathy, C.R., Pearton, S. J., Buyanova, I.A., Rudko, G.Y., Chen, W. M., Pan, C. C., Chen, G. T., Chyl, J I., \& Zavada, J. M. (2004). Electrical and Luminescent Properties and the Spectra of Deep Centers in GaMnN/InGaN Light-Emitting Diodes, Journal of Electronic Material, 33, 241. http://dx.doi.org/10.1007/s11664-004-0186-7

Schubert, E. F. (2006). Light Emitting Diodes. Cambridge University Press, p.75-83. 